\title{
Abnormal response to negative feedback in unipolar depression: evidence for a diagnosis specific impairment
}

\author{
R Elliott, B J Sahakian, J J Herrod, T W Robbins, E S Paykel
}

Department of Experimental Psychology, University of Cambridge,

Downing Street, Cambridge CB2 3EB, UK

R Elliott

$\mathrm{T}$ W Robbins

Department of Psychiatry, University of Cambridge School of Clinical Medicine, Addenbrookes Hospital, Cambridge CB2 2QQ, UK

B J Sahakian

J J Herrod

E S Paykel

Correspondence to: Dr B J Sahakian,

Department of Psychiatry, University of Cambridge

School of Clinical Medicine, Addenbrookes Hospital, Cambridge CB2 2QQ, UK

Received 10 June 1996 and in final revised form 5 March 1997

Accepted 6 March 1997

\begin{abstract}
Objectives-To assess in further detail the specific form of motivational impairment influencing neuropsychological performance in depression-oversensitivity to perceived failure. The present study considers two questions: firstly whether this is specific to depression and secondly how the effect relates to clinical features.

Methods-Unipolar depressed patients and matched controls were assessed on two neuropsychological tests giving explicit performance feedback. The data were analysed in two separate studies to consider the questions above. The first study considered the specificity of the effect to depressed patients, using data on the same tests collected from other patient groups. The second study was a longitudinal assessment of the depressed patients on clinical recovery to determine whether the effect is specific to the depressed state. Results-The effect was not seen in nondepressed patient groups, either neurological or psychiatric groups. The longitudinal study showed a residual abnormal response to negative feedback on clinical recovery.

Conclusions-Abnormal response to negative feedback is specific to a primary diagnosis of depression and may be a trait rather than a state factor of the disorder. These results are discussed in relation to the putative neuropathology of depression and also to cognitive and behavioural accounts of the disorder. The findings presented here have important implications for establishing a link between mood and cognition in unipolar depression.
\end{abstract}

(F Neurol Neurosurg Psychiatry 1997;63:74-82)

Keywords: negative feedback; depression; CANTAB tests; mood and cognition

Numerous studies have shown the presence of wide ranging neuropsychological deficits associated with unipolar depression, ${ }^{1-7}$ but a coherent account of these deficits has proved elusive. One reason for this is that it is not clear to what extent these neuropsychological impairments may be mediated by abnormal psychological mechanisms. In our recent study of unipolar depression, ${ }^{1}$ we identified a specific psychological mechanism which can have a general effect on neuropsychological performance. We de- scribed this mechanism as a "catastrophic response to perceived failure" (after Beats et $a l^{\beta}$ ) and suggested that this effect represents an important link between cognitive and emotional processes affecting the performance of depressed patients.

In depression, various psychological mechanisms closely related to clinical symptoms may influence cognitive function. An often cited example is that many neuropsychological impairments may be due to a more fundamental deficit of motivation. Psychotic patients in general, and depressed patients in particular, do show motivational deficits influencing cognition. ${ }^{8}$ These authors attempted to distinguish between the "computational" and "energetical" (including motivational) aspects of information processing, described by Hockey et $a l^{9}$ and argued that the energetical aspects were impaired in depression. However, Richards and Ruff ${ }^{10}$ have shown that manipulations of motivation do not necessarily affect cognitive performance.

Motivation should not be considered as a unitary concept, rather as one made up of various interrelated components. One of these, and the one which will be considered in the present paper, is the role of reinforcement in controlling behaviour. Many neuropsychological tests give subjects feedback for their responses and there is some evidence to suggest that the response of depressed patients to this feedback is different from that of control subjects. Hughes et $a l^{11}$ showed that depressed patients were less responsive to reward than controls. They used a progressive reinforcement schedule for financial reward and found that depressed subjects worked less for reward and earned less. There is also evidence that subjects judge their performance in a more negative way than controls. Wener and Rehm ${ }^{12}$ gave depressed and non-depressed students either $20 \%$ or $80 \%$ success feedback on a word association task then asked for an estimate of their success rate. Depressed patients reported a lower self assessed success rate than controls. Thus it seems that depressed patients show a bias in their response to reinforcement. It is a reasonable prediction that depressed patients will respond differently to negative feedback than controls, resulting in altered cognitive performance.

In a recent study of depression in elderly people, Beats et $a l^{\beta}$ found that these patients showed what was interpreted as a "catastrophic response to executive failure". On a test of 
planning, the Tower of London test from the CANTAB battery, ${ }^{13}{ }^{14}$ the patients solved as many problems correctly as controls but once they had made a mistake on a problem, their performance deteriorated rapidly. Thus although they solved as many problems within the minimum number of moves as the controls, they solved fewer within the maximum number of moves. This effect suggests a link between psychological and neuropsychological impairments and our recent study of middle aged patients with unipolar depression ${ }^{1}$ examined it more systematically.

We focus here on two tests; the delayed matching to sample test (DMTS) from the CANTAB battery ${ }^{15}$ and a novel one touch Tower of London test ${ }^{17}$ based on the planning task from the CANTAB battery used by Beats et $a l .{ }^{3}$ As discussed in Elliott et al, ${ }^{1}$ the depressed patients showed pronounced deficits on both tasks. On the delayed matching to sample test, patients were significantly impaired under all delay conditions, including the simultaneous condition. On the new Tower of London test, they were significantly impaired at all levels of difficulty.

These tasks have very different neural substrates including temporal lobe structures in the first case ${ }^{18}$ but parietal and frontal regions in the second (based on PET evidence $\left.{ }^{19}\right)$. However, they have certain procedural similarities; both require subjects to process complex stimuli and then make a single choice response from an array of alternatives; both present easier problems interspersed with more difficult ones and, crucially, both give subjects immediate and explicit feedback. Thus subjects are given feedback to one problem and then are immediately required to process the subsequent problem. We found that on both tests, failure on one problem in the depressed subjects significantly raised the probability of failure on the subsequent problem relative to controls. ${ }^{1}$

The present study aimed to develop these findings more fully by introducing data from other patient groups and from a longitudinal study of depression. This study had two specific aims. The first was to determine whether the abnormal response to perceived failure is relatively specific to a diagnosis of depression. It is possible that the effect seen was due to the raised overall failure rate in depressed patients compared with controls simply because exposure to higher rates of negative feedback alters response to it. Secondly, this paper assesses the relation between abnormal response to perceived failure and clinical variables in depression, considering whether it represents a trait or a state factor. These questions are considered in two separate studies.

\section{Study 1}

COMPARISON WITH OTHER PATIENT GROUPS It is important to consider whether the abnormal response to negative feedback is specific to depression. It may be, for example, that a nondepressed patient group who showed impairments on the tests would respond abnormally
Table 1 Characteristics of groups whose performance was analysed on the delayed matching to sample task

\begin{tabular}{llll}
\hline & $\begin{array}{l}\text { Patients } \\
(n)\end{array}$ & Mean age & Mean NART-IQ \\
\hline Depressed & 28 & $48.1(1.2)$ & $111.8(1.7)$ \\
Control & 22 & $50.0(1.7)$ & $113.7(1.3)$ \\
Parkinson's & 17 & $61.1(1.8)$ & $108.2(2.2)$ \\
Temporal lobe & 23 & $34.2(1.6)$ & $107.9(1.7)$ \\
Schizophrenic & 11 & $43.4(3.3)$ & $110.4(3.0)$ \\
\hline
\end{tabular}

Values in parentheses are SEM.

to negative feedback, perhaps because they experience more of it during the tests than controls. The finding may be an artefact due to raised overall failure rates, and therefore may not have any import for the understanding of the neuropsychology of depression. To consider this question, the performance of nondepressed groups with impairments on these tests was considered.

\section{METHOD}

Subjects

Depressed patients-The depressed patients were the 28 inpatients and outpatients described by Elliott et $a l,{ }^{1}$ of whom 19 were women and eight were men. All patients met DSM-III- ${ }^{20}$ criteria for major unipolar depressive disorder, and patients with concurrent diagnoses of psychoactive substance misuse or neurological or general medical disorders likely to affect cognition (for example, dementia, stroke, Parkinson's disease, head injury, untreated thyroid disease) were excluded. Fourteen of the patients were in their first episode of depression and 14 had recurrent depressive disorder. Phenomenology was recorded using the present state examination. Patients were not tested within three months of receiving electroconvulsive therapy. Patients taking antidepressant medication were included, but not those taking neuroleptics or steroids, or those having taken benzodiazepines in the 24 hours before testing. All the patients were taking medication; five clomipramine, four amitriptyline, four dothiepin, one lofepramine, three sertraline, two fluoxetine, two paroxetine, two moclobemide, two lithium with dothiepin, one lithium with amitriptyline, and one lithium with phenelzine.

Control subjects - The 22 control subjects (as described by Elliott et $a l^{1}$ ) matched the patients for age and premorbid IQ (as measured by the national adult reading test, NART $^{21}$ ) (tables 1 and 2). Fifteen $(68.2 \%)$ of the control subjects were women and seven were men. Control subjects were excluded if they showed any evidence of psychiatric or neurological history, substance misuse, or were taking any concurrent medication which could influence cognition. The patients and controls did not differ in terms of male:female ratio, or in terms of age or NART-IQ, as shown by unpaired $t$ tests $(t=0.63, \mathrm{P}>0.25$; and $t=1.06, \mathrm{P}>0.5$ respectively).

Groups of neurological patients and intellectually intact patients with schizophrenia (all scoring above 85 on the WAIS-R and with current IQs within 10 points of premorbid IQs) who had been tested on the DMTS and Tower 
Table 2 Characteristics of groups whose performance was analysed on the new Tower of London task

\begin{tabular}{llll}
\hline & $n$ & Mean age $(y)$ & Mean NART-IQ \\
\hline Depressed & 28 & $48.1(1.2)$ & $111.8(1.7)$ \\
Control & 22 & $50.0(1.7)$ & $113.7(1.3)$ \\
Parkinson's & 15 & $59.8(1.7)$ & $109.6(2.2)$ \\
Frontal lobe & 12 & $50.9(3.6)$ & $114.7(2.8)$ \\
Schizophrenic & 12 & $43.4(3.3)$ & $110.4(3.0)$ \\
\hline
\end{tabular}

Values in parentheses are SEM.

of London tasks were selected to match the depressed group for overall performance on the specific tests rather than for age or NART-IQ. For this purpose there was a strong rationale for using respectively patients with frontal lobe excision for making comparisons on the Tower of London test and patients with temporal lobe excision for making comparisons on the DMTS test as these two groups show selective impairments on the two tests. ${ }^{17} 18$ We also selected two relevant neuropsychiatric groups for comparison with the depressed patients who exhibit deficits on both tasks-patients with Parkinson's disease ${ }^{17} 22$ or schizophrenia. ${ }^{23}$ The comparison with Parkinson's disease is important because it represents a form of "subcortical (or frontosubcortical) dementia" 24 and depression has also been considered in the same category on the grounds of overlap in clinical features and possible neural substrates, including basal ganglia pathology. ${ }^{25}$ The comparison with schizophrenia is informative because it represents a distinct form of psychosis, with likely pathology in the temporal lobe, as well as in frontostriatal circuitry. ${ }^{27}{ }^{28}$ Full details of the neurological groups, including basic neuropsychological performance, have been published recently. ${ }^{17}{ }^{18} 22{ }^{23}$ Tables 1 and 2 show the demographic characteristics and overall performance of these patients.

\section{Procedure}

Clinical evaluation - Severity of depression was assessed with the Hamilton depression scale $\left(\right.$ Ham-D ${ }^{29}$ ) and the Montgomery Asberg depression rating scale $\left(\mathrm{MADRS}^{30}\right)$. Mean scores of the patient group were as follows: Ham-D 22.4 (SD 0.8), range 15 to 35; MADRS 34.1 (SD 1.1), range 25 to 46 .

Neuropsychological evaluation-Patients were assessed on a battery of computerised neuropsychological tests including core tests from the CANTAB battery, which has been discussed in detail elsewhere (for example, Robbins $\left.e t a l^{16}\right)$. Results from the full battery were discussed in our recent paper. ${ }^{1}$ For the purposes of the present discussion, two tests are of particular relevance.

Simultaneous and delayed matching to sample-This test has been described in detail elsewhere. ${ }^{16}$ Subjects were presented with a complex abstract pattern and then asked to pick this pattern from a choice of four similar stimuli. In simultaneous matching to sample (MTS) the sample stimulus remained on the screen while choice stimuli appeared. In the delayed matching to sample (DMTS) task, the sample stimulus disappeared before the choice stimuli were presented after delays of 0,4 , or 12 seconds. The MTS and different DMTS delays were presented in a randomised order, the same order for each subject.

New Tower of London task-(see Owen et al ${ }^{17}$ for a full description) Subjects were presented with two arrays of three coloured balls and were asked to work out, using specified rules, the minimum number of moves it would take to achieve the goal arrangement. This differs from earlier Tower of London tasks in that it requires subjects to mentally manipulate the arrays without physically moving the balls. There were a total of 20 problems of one to five moves, presented in a random order, the same order for each subject.

\section{Data analysis}

As discussed in Elliott et $a l^{1}$ various post hoc analysis methods could be used to assess whether subsequent performance is influenced by feedback. The method we chose was one of the simplest, which considered the "conditional probability" of a subject failing a problem in the DMTS or new Tower test given that they had failed the directly preceding problem. Conditional probabilities are usually calculated by Bayes' theorem, which states that the probability of event $\mathrm{B}$ occurring given that event $\mathrm{A}$ has already occurred (denoted $\mathrm{p}(\mathrm{A}$ I B)) is the probability of both events occurring $(\mathrm{p}(\mathrm{AB})$ ) divided by the probability of $A$ occurring. If events $A$ and $B$ are independent, this will be the same as the probability of B occurring.

In the present analysis, event $\mathrm{A}$ is failing a problem, say problem $\mathrm{x}$, and event $\mathrm{B}$ is failing the subsequent problem, problem $x+1$. Therefore the conditional probability is:

$\mathrm{p}(\mathrm{x}+1$ wrong $1 \mathrm{x}$ wrong $)=\frac{\mathrm{p}(\mathrm{x} \text { and } \mathrm{x}+1 \text { wrong })}{\mathrm{p}(\mathrm{x} \text { wrong })}$

This is equivalent to dividing the number of errors made immediately after another error by the total number of errors made by that subject. As the calculation involves dividing by the overall probability of failure, the difference in overall failure rate is controlled for. If, for example, a patient failed twice as many problems as a control (say $2 \mathrm{x}$ as opposed to $\mathrm{x}$ ), and if there was no difference in the effect of failure on subsequent performance they would be expected to fail twice as many problems after other failed problems as controls (say $2 \mathrm{y}$ as opposed to $\mathrm{y}$ ). Thus the conditional probability for patients would be $2 \mathrm{y} / 2 \mathrm{x}$ which is exactly the same as that for controls; $y / x$. This method of analysing the data would be inappropriate if the performance of the patient group was approaching floor level; however, all the patients were performing well above this.

Having calculated this probability for all subjects, univariate $F$ tests were used to compare the values for depressed patients and the other patient groups. These were calculated using the statistical package for social sciences $\left(\mathrm{SPSS}^{26}\right)$.

\section{RESULTS}

Effects of negative feedback

As described in detail in Elliott et al, ${ }^{1}$ depressed patients solved fewer problems correctly than controls in both the tests (fig 1A). Conditional 

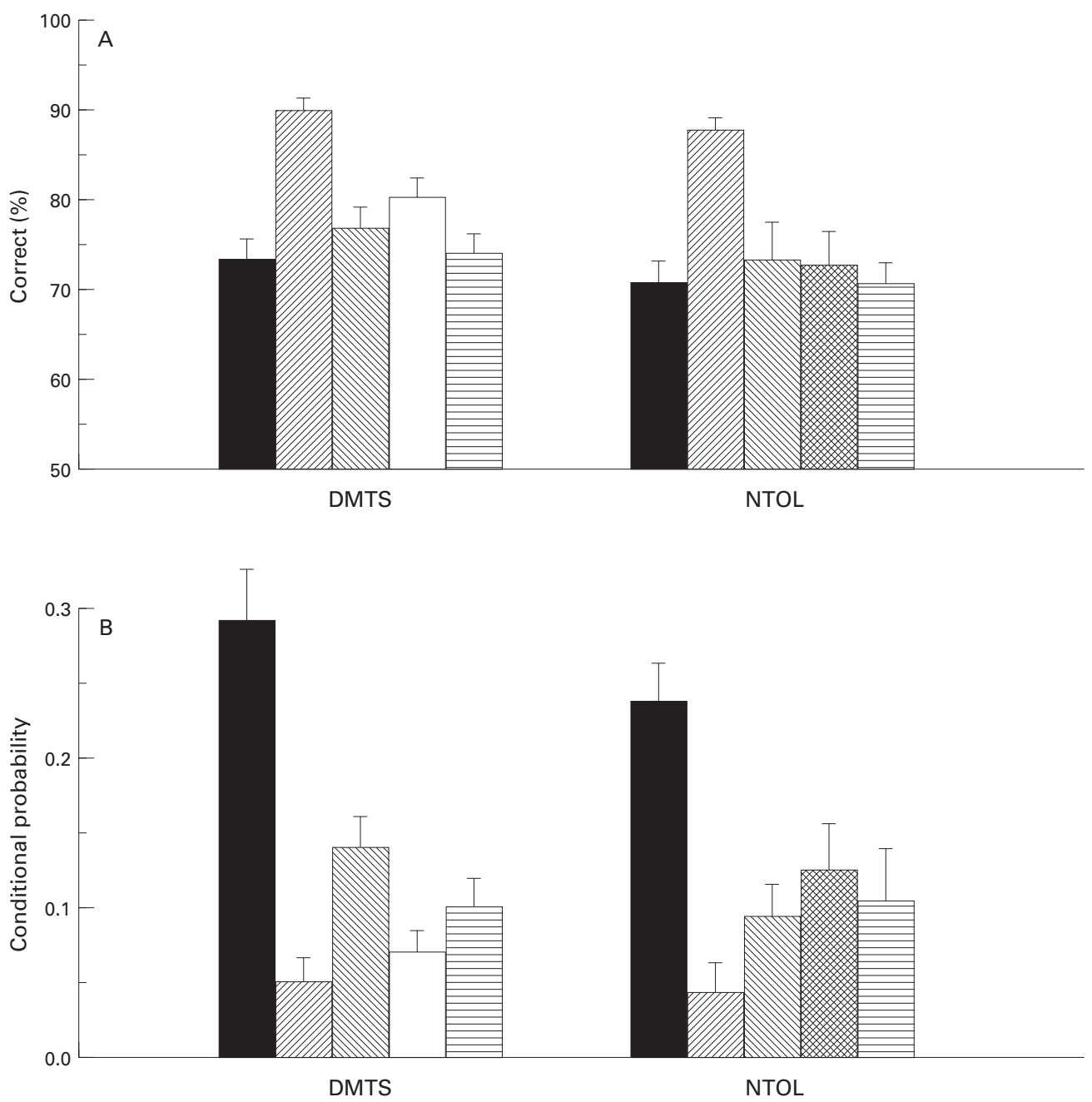

Figure 1 The percentage of problems solved correctly $(A)$ and conditional probabilities of failing problem $x+1$ having just failed problem $x$ (B) on the DMTS and one touch Tower tests for depressed patients, controls, patients with Parkinson's disease, neurosurgical patients, and patients with schizophrenia. Bars represent SEM.

probabilities of failing a problem having failed the preceding problem are shown in the relevant columns of fig $1 \mathrm{~B}$. When these probabilities were compared, there was a significant main effect of group, both on $\operatorname{DMTS}(F(1,48)=25.0, \mathrm{P}<0.001)$ and on new Tower $(F(1,48)=19.5, \mathrm{P}<0.001)$. For the patient group these conditional probabilities for the two tests correlated significantly with each other $(r=0.53, \mathrm{P}<0.01)$.

Comparison with non-depressed groups

For the overall percentage correct score on the DMTS task, one way, between subjects analysis of variance (ANOVA) was performed on the scores of the depressed patients, controls, patients with Parkinson's disease, patients with temporal lesions, and schizophrenia. The analysis showed that there was a significant main effect of group $(F(4,97)=9.7, \mathrm{P}<0.001)$. Post hoc Newman-Keuls tests showed that this effect was due to all four patient groups differing from controls at the $\mathrm{P}<0.01$ level of significance but not differing from each other. Thus the patient groups matched for overall level of impairment relative to controls (fig 1A).

Figure 1B shows the conditional probabilities for these five groups of failing a DMTS problem given that they had failed the preceding problem. One way ANOVA showed a significant main effect of group $(F(4,97)=8.5$, $\mathrm{P}<0.001)$. Post hoc Newman-Keuls tests showed that this effect was due to the depressed patients differing from the other four groups at the 0.01 significance level. The other patient groups did not differ significantly from the controls.

For the overall percentage correct score on the new Tower of London task, a one way, between subjects ANOVA performed on the scores of the depressed patients, controls, patients with Parkinson's disease, patients with frontal lobe lesions, and patients with schizophrenia. This analysis showed that there was a significant main effect of group $(F(4,84)=4.7$, $\mathrm{P}<0.01)$. Post hoc Newman-Keuls tests showed that this effect was due to all four patient groups differing from controls at the $\mathrm{P}<0.05$ level of significance but not differing from each other. Thus the patient groups were roughly matched for overall level of impairment (fig 1A).

Figure 1B shows the conditional probabilities of failing a new Tower problem given that they had failed the preceding problem for these 
four groups. One way ANOVA showed a significant main effect of group $(F(4,84)=3.7$, $\mathrm{P}<0.005)$. Post hoc Newman-Keuls tests showed that this effect was due to the depressed patients differing from the other four groups at the 0.05 significance level. The other patient groups did not differ from the controls.

These analyses show that the abnormal response to negative feedback is relatively specific to depression. They clearly show that the effect is not simply an artefact due to patients failing more problems overall, as other groups of patients matched for overall level of impairment do not show the same effect. It also seems that the abnormal response to negative feedback is not a general trait of psychiatric patients, as the effect was not seen in the patients with schizophrenia, but is specific to depression.

\section{Study 2}

RELATIONS BETWEEN ABNORMAL RESPONSE TO NEGATIVE FEEDBACK AND CLINICAL VARIABLES Given that the abnormal response to negative feedback is an influence on performance which seems to be specific to a diagnosis of depression, it is important to determine how it may relate to clinical symptoms. In particular, the effect may be either a state or a trait factor of the disorder.

\section{METHODS}

Subjects

Depressed patients on clinical recovery-In the follow up stage of this longitudinal study, the patients in the group described above were monitored after the initial test session until they were judged to be clinically recovered by a consultant psychiatrist. Additionally, clinical recovery was defined as a score of 8 or less on the Hamilton depression scale. Of the 28 patients originally studied, one committed suicide, two were lost to follow up, two withdrew from the study, and nine did not recover within the available time scale (most showed improvement but did not fall within the defined range for clinical recovery). This left 14 patients who did recover, of whom 10 were female and four were male. These patients were retested between five and 18 months (mean 12.6 months) after the initial test session. The mean age of these patients was 47.9 (SEM 1.5) and their mean NART-IQ was 110.7 (2.5). As currently accepted practice is for patients to continue to take medication for at least six months after recovery and then to withdraw gradually, these patients were still taking antidepressant medication at the time of follow up.

Control subjects-Fourteen of the original controls described in Elliott et al , ${ }^{1} 10$ women and four men to match the patients, were retested between six months and two years (mean 14.1 months) after the initial test session, such that the mean time elapsed between test sessions was the same for both groups. The mean age of these controls was 50.2 (SEM 2.3) and their mean NART-IQ was 114.5 (SEM 1.6). Univariate $F$ tests showed that patients and controls did not differ in terms of age or IQ.

\section{Procedure and data analysis}

Clinical evaluation-The recovered patients were assessed with the Hamilton depression scale $^{29}$ and the Montgomery Asberg depression rating scale. ${ }^{30}$ Mean scores were 3.0 (SEM 0.75) on the Ham-D and 5.1 (SEM 2.3) on the MADRS.

Neuropsychological evaluation and data analysis-Subjects completed the same test battery as at initial assessment, including the DMTS and Tower of London tasks. Response to perceived failure was assessed by analysing conditional probabilities as described above. For simplicity the conditional probabilities on the two tests were summed to give an overall "response to negative feedback score". These scores, together with percentage correct scores, for patients and controls were compared using repeated measures ANOVA.

RESULTS

Follow up of patients on clinical recovery

Figure 2A shows the overall percentages correct on the DMTS and Tower of London tests. Repeated measures ANOVA of the DMTS scores showed significant effects of group $(F(1,26)=10.8, \mathrm{P}<0.01)$ and test session $(F(1,26)=5.7, \mathrm{P}<0.05)$. Depressed patients performed worse overall than controls and both groups performed better on the second session than the first. There was also a near significant group $\mathrm{x}$ test session interaction $(F(1,26)=4.1, \mathrm{P}=0.054)$ due to the improvement in patients' performance on clinical recovery, which would probably reach significance in a larger sample of patients. Repeated measures ANOVA of the Tower of London scores showed a significant main effect of group $(F(1,26)=9.1, \mathrm{P}<0.01)$, with patients solving fewer problems than controls. There was also a significant group $\mathrm{x}$ test session interaction $(F(1,26)=5.1, \mathrm{P}<0.05)$ which simple main effects analysis showed was due to patients performing much less accurately than controls at initial assessment $(F(1,26)=10.9$, $\mathrm{P}<0.01)$ and to patients performing significantly better on clinical recovery than when depressed $(F(1,26)=9.3, \mathrm{P}<0.01)$.

Figure $2 \mathrm{~B}$ shows the conditional probabilities of failing problem $x+1$ having failed problem $\mathrm{x}$ on the DMTS and Tower of London tests at initial assessment and follow up. These probabilities were summed to give a total "response to negative feedback score" as described above. Two way repeated measures ANOVA showed only a significant main effect of group $(F(1,26)=13.3, \mathrm{P}<0.01)$. Figure $2 \mathrm{~B}$ shows a trend towards improvement on clinical recovery and in a larger group of patients this may have reached significance; however, the crucial finding is that clinically recovered patients are still impaired relative to controls. It should also be noted that recovered patients show abnormal response to negative feedback on the new Tower of London test even though their performance level was not significantly different from that of controls. This is further 

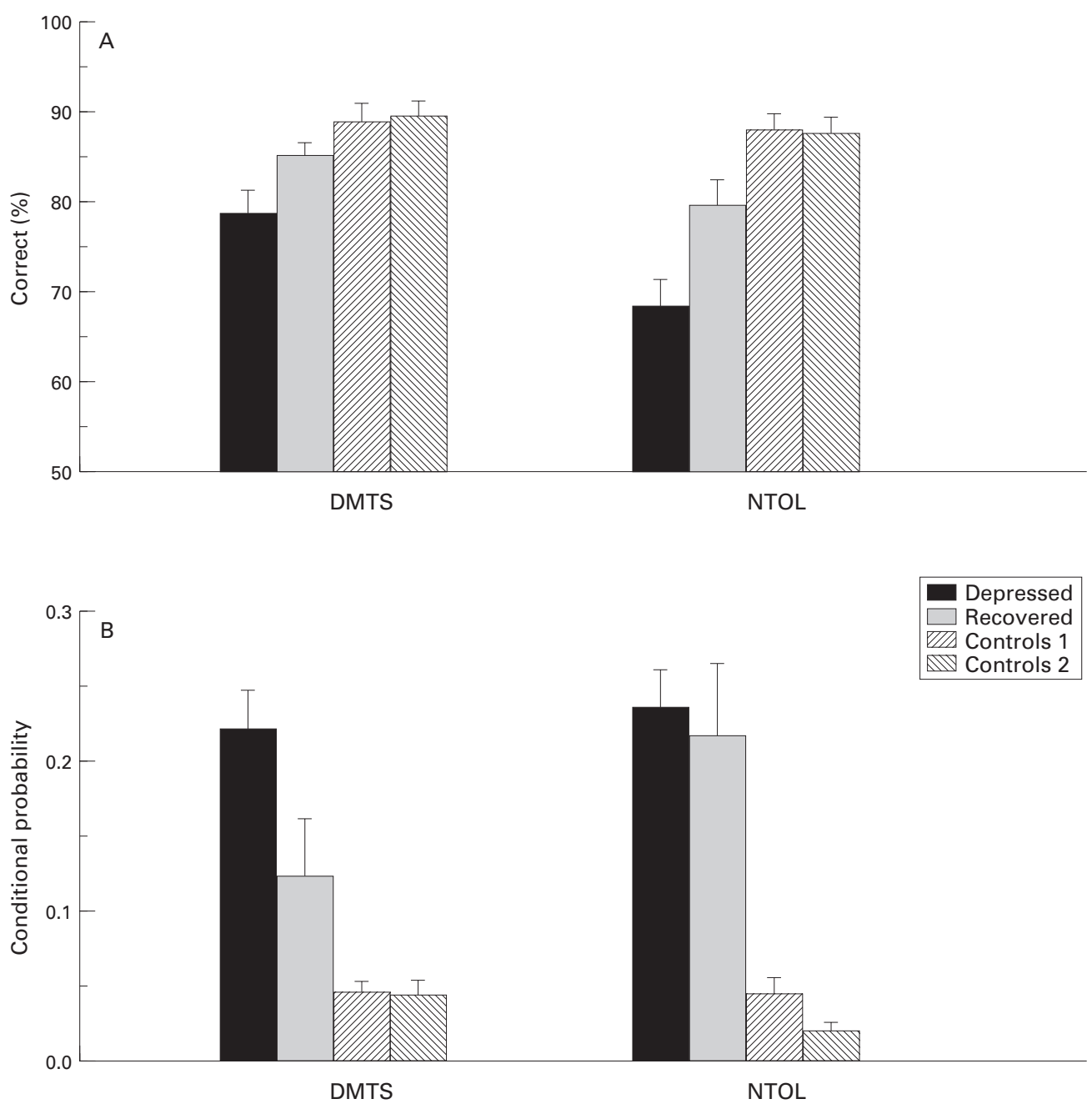

Figure 2 (A) The mean total percentages correct for the depressed patients at initial assessment and on recovery with control patients tested across two sessions on the DMTS and one touch Tower tasks. (B) The corresponding mean conditional probabilities of failing problem $x+1$ having failed problem $x$. Bars represent SEM.

evidence that raised conditional probability of subsequent failure is not simply a consequence of a higher overall failure rate.

The Pearson product moment correlation coefficients were calculated between the response to failure score and the recovered scores on the Ham-D and MADRS clinical rating scales to assess whether these residual impairments may be related to residual depression. These correlations were not significant at the $\mathrm{P}<0.05$ level.

\section{Comparison of recovered with non-recovered} patients

It is possible that clinical recovery is predicted by a different pattern of neuropsychological performance while depressed. To study this, the performance (at initial assessment, not on recovery) of the 14 patients who did recover in the time available was compared with that of the nine who did not. A one way ANOVA was carried out with group (recovered $v$ nonrecovered) as the between subject variable and delay or difficulty level as the within subject variable where appropriate. There was a significant difference between the total response to negative feedback score of the two groups $(F(1,21)=4.72, \mathrm{P}<0.05)$, with the group who subsequently recovered showing a less abnormal response than the group who did not recover, with total scores of 0.39 (SEM 0.07 ) and 0.66 (SEM 0.1) respectively.

Comparison between first episode and recurrent depression

There was no significant difference between the total response to failure scores of patients with first episode depression and those with recurrent depression (0.59 (SEM 0.1) and 0.49 (SEM 0.08 respectively, $F(1,26)=1.7$, $\mathrm{P}>0.05)$.

\section{Discussion}

The results of this study strongly suggest that one of the general processes influencing cognitive performance in depressed patients is a highly specific form of deficit involving an abnormal response to negative feedback. Our previous study ${ }^{1}$ explicitly showed, we think for the first time, that failure on problem ( $\mathrm{x}$ ) dramatically increases the chance of failure on problem $(x+1)$ in depressed patients relative to controls. The present study shows, crucially, that this effect is specific to depression within 
the context of the comparison groups used here. Other pertinent patient groups with the same overall failure rate did not show the same effect. This shows firstly, that the effect cannot be an artefact of the raised failure rates and secondly, that the effect is relatively specific to depression. Further evidence that the effect is not an artefact due to raised failure rate is that it remained significant in clinically recovered patients whose overall performance level had improved. Although there was a strong trend for response to negative feedback to improve on clinical recovery, it remained significantly different from controls. This residual abnormality suggests that the effect may be a trait rather than a state factor for depression. However, it might simply reflect the greater sensitivity of this state measure over others. It would be interesting, therefore, to pursue the trait hypothesis in a prospective study.

It should be noted that the present study has not assessed whether the altered response to negative feedback is accompanied by an abnormal response to positive feedback. An analogous method of analysis to that described in Elliott $e t a l,{ }^{1}$ and used here, to assess the effect of negative feedback would not be appropriate for analysing the effects of positive feedback as all the subjects studied were scoring more than $50 \%$ correct.

Whereas the present study has shown unequivocally an abnormal response to negative feedback relatively specific to depression, the precise psychological mechanisms underlying this effect have not been clearly characterised. Feedback potentially has both cognitive and emotional sequelae, the second of which could either improve or disrupt performance. Subjects can use negative feedback to monitor and improve their performance but this cognitive component to their response is accompanied by emotional reactions. This may take the form of an increased determination to succeed, motivated by a desire to achieve positive reinforcement or it may take the form of a more negative reaction such that subsequent performance is disrupted. The overall response of subjects to negative feedback may depend on a subtle interplay of these positive and negative factors. Previously, we suggested that the abnormal response in the depressed group may represent an "oversensitivity to negative feedback". The resultant disruptive effect on subsequent cognitive performance has been described as a "catastrophic response to failure". This account suggests that the negative emotive response to feedback on incorrect trials impairs performance in depressed patients.

However, closer scrutiny of the data suggests that an alternative explanation of the effect may also be viable. Thus when the conditional probability of failure measure relative to the overall probability of failure is considered, the evidence suggests that it may be the control subjects who show a greater response to negative feedback. Specifically, their mean conditional probability of failure $(0.03$, fig 1$)$ is numerically lower than their average probability of failure (mean percentage correct $90 \%$, fig 1, therefore probability of failure, 0.1). By contrast, these two measures are much more similar in the depressed group (conditional probability of failure 0.26, average probability of failure $0.23)$. Therefore these results could be interpreted as reflecting an unaltered response to negative feedback in the depressed group relative to controls and to non-depressed patient groups. In this study, control subjects apparently responded to negative feedback by reducing errors on the subsequent trials. This effect could therefore represent either the enhanced use of knowledge of results to monitor and improve performance, an increased motivation to respond for positive reinforcement, or a combination of these cognitive and emotive factors. There is some evidence to support this account from response latencies to problems after a failed problem. In control subjects, these response latencies showed a strong trend to be significantly longer than overall response latencies $(\mathrm{P}<0.08)$ to problems of the same level of difficulty. In depressed patients, by contrast, these latencies were not significantly different from overall response latencies. Whereas it is therefore clear that patients were not "giving up" and making rapid guesses, they were not taking longer over problems to improve their performance.

The abnormal response to negative feedback in depressed patients may thus reflect inefficiency in using knowledge of results to monitor performance. Alternatively it may represent a motivational impairment such that patients are less motivated by potential reward than controls and so do not experience the same determination to improve performance in the light of negative feedback. It is also possible that both these factors are involved in the abnormality; indeed, they may be interdependent. Using the cognitive information provided by negative feedback to monitor and improve performance may depend on subjects being motivated to achieve positive rather than negative feedback.

There are thus two different explanations of the findings presented in this paper; an impairment of performance in response to negative feedback in depressed patients representing an adverse emotive reaction, or an enhancement of performance in controls representing a possible interaction of cognitive and emotional effects. Of course, these explanations are not necessarily mutually exclusive and it is entirely plausible that both are contributing to the overall effect. Further studies are necessary to consider these hypotheses explicitly; however, what can be concluded unequivocally from the present data is that the response to negative feedback is abnormal in patients with depression.

The abnormal response to negative feedback can be seen as an important link between mood and performance in depression. Many previous attempts to link these dimensions of dysfunction have relied on the use of emotionally toned information in neuropsychological tasks. The effect described here is a psychological effect related to depressed mood which can influence the processing of neutral material. Suggesting a 
critical role for reinforcement in the performance of depressed patients has much in common with behavioural theories of depression. Lewinsohn et $a l^{\beta 2}{ }^{33}$ proposed an account of depression based on reinforcement, stressing that depressed patients experience and expect low rates of positive reinforcement. The importance of response to feedback in depression can also be linked to cognitive accounts of the disorder. An influential cognitive theory of depression has been developed by $\mathrm{Beck}^{34-36}$ and modified by Teasdale and colleagues. ${ }^{37-39}$ In brief, this theory argues that people vulnerable to depression are characterised by enduring dysfunctional schemata made up of negative attitudes and assumptions. A role for oversensitivity to negative feedback is consistent with these cognitive accounts. Patients may be distracted by internal ruminations or by external stimuli with a detrimental effect on subsequent performance. Their bias towards processing the negative feedback may detract from their processing of the subsequent problem. Alternatively, or additionally, the negative attitudes and assumptions of depressed patients may compromise their ability or motivation to improve subsequent performance in the light of negative feedback.

Abnormal responses to negative feedback are therefore consistent with cognitive and behavioural accounts of depression and may prove an important component underpinning both neuropsychological and clinical aspects of the disorder. The comparisons with other patient groups described here suggest that the effect may be relatively specific to depression rather than being a general consequence of raised failure rates or an artefact of the chosen method of statistical analysis. When groups of non-psychiatric patients with a similar overall level of impairment were compared with the depressed patients, they did not show the same effect of oversensitivity to negative feedback. The conditional probabilities of failing a problem having just failed the preceding problem did not differ significantly from controls for the patients with severe Parkinson's disease or neurosurgical patients with frontal or temporal lobe lesions. Although these groups failed more problems overall than the controls, the same proportion of incorrect responses followed other incorrect responses. This is an important result in the light of the debate surrounding the extent to which impairments associated with Parkinson's disease can be attributed to co-occurring depression. ${ }^{40-42}$ The present findings suggest that there are qualitative differences in the performance of the two groups which means that several of the deficits found in patients with Parkinson's disease cannot be attributed to depression. The abnormal response to negative feedback was also absent in schizophrenic patients, suggesting that it is not a phenomenon which is common to psychiatric disorders but one which is relatively specific to depression. Interestingly, one of our explanations of the effect as a monitoring deficit might predict a similar impairment in patients with schizophrenia, a disorder hypothesised to be associated with monitoring impairments. ${ }^{43}$
However, no such deficit was seen in this high functioning patient group.

In neural terms, the possible existence of a general factor influencing performance of cognitive tasks that engage probably very different neocortical networks may implicate neuromodulatory function of those ascending neurotransmitter pathways innervating wide areas of the neocortex such as noradrenergic and serotonergic systems, which are, of course, already associated with depression. ${ }^{44}{ }^{45}$ Another possible neural substrate for the effect is in the frontal cortex. Damasio ${ }^{46}$ described in detail a patient who had extensive damage to the prefrontal cortices, particularly the ventromedial regions. This patient showed relatively preserved neuropsychological function but was impaired at decision making, due to an inability to attach emotional salience to situations. This impairment has clear parallels with the suggested abnormal emotional response to feedback in depressed patients and an involvement of orbitofrontal pathology in the second effect could therefore be hypothesised.

Regardless of what the neural substrate of the effect might be, it is clear that response to negative feedback has a significant influence on neuropsychological performance. Obviously, this effect is likely to be most striking in tests where immediate and explicit feedback is given after each problem. However, even in tests in which this is not the case, subjects can usually form an impression of how well they are performing. Whereas the effect cannot be explicitly measured in such tests, it may still be occurring. If oversensitivity to negative feedback plays a part in the abnormality in depressed patients, this would be further exacerbated if, as predicted by cognitive and behavioural accounts, they are more self critical than controls and are thus more likely to view their performance as inadequate.

The finding that abnormal response to failure may be more closely related to whether depressed patients are admitted to hospital even than severity of depression as measured on clinical scales, ${ }^{1}$ provides evidence for the clinical validity of the measure which may also have implications for the behavioural management and treatment of depression. The finding of study 2 , that clinically recovered depressive patients still show an abnormal response to negative feedback is also consistent with the findings of Vaughn and Leff ${ }^{47}$ that high levels of criticism increased relapse rate in depression.

In conclusion, the abnormal response to negative feedback described in this paper represents an advance in our understanding of depression and potentially provides a crucial link between mood and performance. The finding is consistent with cognitive and behavioural accounts of the disorder and may have implications for treatment. Further studies are needed to replicate this effect independently, perhaps in comparison with other forms of affective disorder-for example, generalised anxiety, obsessive-compulsive disorder, and bipolar depression-and characterise it more fully in cognitive terms. 
This work was funded by a Wellcome Trust Programme Grant to Drs TW Robbins, BJ Everitt, AC Roberts, and BJ Sahakian. RE was funded by a Wellcome Trust prize studentship. We thank Drs Adrian Owen, Peter McKenna, and Paula McKay for their contribution to studies from which these data were obtained. ${ }^{1171822} 23$

1 Elliott R, Sahakian BJ, McKay AP, Herrod JJ, Robbins TW, Paykel ES. Neuropsychological impairments in unipola depression: the role of perceived failure on subsequent performance. Psychol Med 1996;26:975-89.

2 Austin M-P, Ross M, Murray C,O'Carroll RE, Ebmeier KP, Goodwin GM. Cognitive function in major depression. $\mathscr{f}$ Affect Disord 1992;25:21-30.

3 Beats BC, Sahakian BJ, Levy R. Cognitive performance in tests sensitive to frontal lobe dysfunction in the elderly depressed. Psychol Med 1996;26:591-603.

4 Brown RG, Scott LC, Bench CJ, Dolan RJ. Cognitive function in depression: its relationship to the presence and severity of intellectual decline. Psychol Med1994;24:82947.

5 Golinkoff M, Sweeney JA. Cognitive impairment in depression. F Affect Disord 1989; 17:105-12.

6 Miller WR. Psychological deficit in depression. Psychol Bull 1975;82:238-60.

7 Weingartner $\mathrm{H}$, Cohen RM, Martello JD, Gerdt C. Cognitive processes in depression. Arch Gen Psychiatry 1981;38:42-7.

8 Schmand B, Kuipers T, Van der Gaag M, M, Bosveld J, Bulthuis F, Jellema $M$. Cognitive disorders and negative symptoms as correlates of motivational deficits in psychotic patients. Psychol Med 1994;24:869-84.

9 Hockey GRJ, Giallard A, Coles MGH. Energetics and human information processing. Dordrecht: Nijhoff, 1986.

10 Richards PM, Ruff RM. Motivational effects on neuropsychological functioning: comparison of depressed $\mathrm{v}$ non depressed individuals. $\mathcal{F}$ Consult Clin Psychol 1989;57:396402 .

11 Hughes JR, Pleasants CN, Pickens RW. Measurement of reinforcement in depression: a pilot study. F Behav Ther Exp Psychiatry 1985;16:231-6.

12 Wener AE, Rehm L. Depressive affect: a test of behavioral hypotheses. F Abnorm Psychol 1975;84:221-7.

13 Owen AM, Downes JD, Sahakian BJ, Polkey CE, Robbins TW. Planning and spatial working memory following fron-

14 Robbins TW, James M, Lange KW, Owen AM, Quinn NP, Marsden CD. Cognitive performance in multiple system atrophy. Brain 1992;115:271-91.

15 Sahakian BJ, Morris RG, Evenden JL, Heald A, Levy R, Philpot M, Robbins TW. A comparative study of visuospatial memory and learning in Alzheimer-type dementia and Parkinson's disease. Brain 1988;111:695-718.

16 Robbins TW, James M, Owen AM, Sahakian BJ, McInnes L, Rabbitt P. Cambridge neuropsychological battery (CANTAB): a factor analytic study of a large sample of normal elderly volunteers. Dementia 1994;5:266-81.

17 Owen AM, Sahakian BJ, Hodges JR, Summers BA, Polkey CE, Robbins TW. Dopamine-dependent frontostriatal planning deficits in early Parkinson's disease. Neuropsychology 1995;9:126-40.

18 Owen AM, Sahakian BJ, Semple J, Polkey CE, Robbins TW. Visuo-spatial short term recognition memory and learning after temporal lobe excisions, frontal lobe excisions or amygdalo-hippocampectomy in man. Neuropsychologia 1995;33:1-24.

19 Baker SC, Rogers RD, Owen AM, Frith CD, Dolan RJ, Frackowiak RSJ, Robbins TW. Neural systems engaged by planning: a PET study of the Tower of London task. Neuropsychologia 1996;6:515-26.

20 American Psychiatric Association. Diagnostic and statistical manual of mental disorders, DSM III-R. APA, 1987.

21 Nelson HE. National adult reading test (NART): test manual. Windsor: NFER-Nelson, 1982 .
22 Owen AM, Roberts AC, Hodges JR, Summers BA, Polkey CE, Robbins TW. Contrasting mechanisms of attentional set-shifting in patients with frontal lobe damage or Parkinon's disease. Brain 1993;116:1159-75.

23 Elliott R. Neuropsychological deficits in a small group of schizophrenic patients with preserved intellectual function. $[\mathrm{PhD}$ thesis.] Cambridge; University of Cambridge.

24 Cummings JL. Subcortical dementia: neuropsychology, neuropsychiatry, and pathophysiology. $\mathrm{Br} f \mathrm{f}$ Psychiatry 1986;149:682-97.

25 Baxter LR, Phelps ME, Mazziotta J, Schwartz J, Gerner R, Selin C, Sumida R. Cerebral metabolic rates for glucose in mood disorders. Arch Gen Psychiatry 1985;42:41-7.

26 Drevets WC, Videen TO, Price JL, Preskorn SH, Carmichael ST, Raichle ME. A functional anatomical study of unipolar depression. F Neurosci 1992;12:3628-41.

27 Robbins TW. The case for frontostriatal dysfunction in schizophrenia. Schiz Bull 1990;16:391-402.

28 Crow TJ. Temporal lobe asymmetries as the key to the aetiology of schizophrenia. Schizophr Bull 1990;16:433-43.

29 Hamilton M. A rating scale for depression. 7 Neurol Neurosurg Psychiatry 1960;23:56-62.

30 Montgomery SA, Asberg M. A new depression scale designed to be sensitive to change. $\mathrm{Br} \mathcal{F}$ Psychiatry 1979; 134:382-9.

31 Nie NH, Hadlai Hull C, Jenkins JG, Jenkins JG, Steinbrenner K, Bent DH. Statistical package for the social sciences. New York: McGraw-Hill, 1970.

32 Lewinsohn PM, Youngren MA, Grosscup SJ. Reinforcement and depression. In: RA Depue, ed. The psychobiology of depressive disorders. New York: Academic Press, 1979. 291-316.

33 Lewinsohn PM. A behavioural approach to depression. In: RJ Friedman, MM Katz, eds. The psychology of depression: contemporary theory and research. New York: Winston and Wiley, 1974:157-185.

34 Beck AT. Depression: clinical, experimental and theoretical aspects. New York: Harper and Row, 1967

35 Beck AT. Cognitive therapy and the emotional disorders. New York: International Universities Press, 1976

36 Beck AT. Cognitive therapy of depression. New York: J Wiley, 1979.

37 Teasdale JD. Cognitive vulnerability to persistent depression. Cognition and Emotion 1988;2:247-74.

38 Teasdale JD, Barnard PJ. Affect, cognition and change. Hove: Lawrence Erlbaum Associates, 1993.

39 Teadale JD, Dent J. Cognitive vulnerability to depression: an investigation of two hypotheses. Br f Clin Psychol 1987;26: 113-26.

40 Rogers D, Lees AJ, Smith E, Trimble M, Stern GM. Bradyphrenia in Parkinson's disease and psychomotor retardation in depressive illness. An experimental study. Brain 1987;110:761-76.

41 Starkstein SE, Prezios TJ, Berthier ML, et al. Depression and cognitive impairment in Parkinson's disease. Brain 1989;112:1141-53.

42 Taylor AE, Saint-Cyr JA, Lang AE, et al. Parkinson's disease and depression: a critical evaluation. Brain 1986;109:27992.

43 Frith CD. The cognitive neuropsychology of schizophrenia. Hove: Lawrence Erlbaum Associates, 1992.

44 Zubenko GS, Moossy J, Koppu M. Neurochemical correlates of major depression in primary dementia. Arch Neurol 1990;47:209-14.

45 Deakin JFW. Amine mechanisms in anxiety and depression. In: JM Elliott, DJ Heal, CA Marsden, eds. Experimental approaches to anxiety and depression. New York: J Wiley, 1992:233-43.

46 Damasio AR. Descartes' error. New York: G Putnam, 1994.

47 Vaughn C, Leff J. The influence of family life and social factors on the course of psychiatric illness. Br f Psychiatry 1976;129:125-37. 